\title{
Two-component pore formation by the novel CDCL proteins ALY short and ALY long from Elizabethkingia anophelis
}

\author{
Bronte A. Johnstone ${ }^{1}$, Sara L. Lawrence ${ }^{2}$, Craig J. Morton ${ }^{1}$, Jordan C. Evans ${ }^{3}$, Michelle P. Christie ${ }^{1}$, \\ Rodney K. Tweten ${ }^{3}$, and Michael W. Parker ${ }^{1,2}$ \\ ${ }^{1}$ Department of Biochemistry and Molecular Biology, Bio21 Molecular Science and Biotechnology Institute, University of Melbourne, \\ Parkville, VIC, 3010, Australia \\ ${ }^{2}$ Structural Biology Laboratory, St. Vincent's Institute of Medical Research, Fitzroy, VIC 3065, AustraliaișEp: \\ ${ }^{3}$ Department of Microbiology and Immunology, University of Oklahoma, Health Sciences Center, Oklahoma City, OK 73104, USA \\ bjohnstone@student.unimelb.edu.au
}

Cholesterol-dependent cytolysins (CDCs) are bacterial pore-forming toxins that are secreted as soluble monomers and oligomerise into large circular pre-pores on the surface of cholesterol-rich membranes. Various structural changes and transitions results in insertion of $\beta$-hairpins into the lipid bilayer, forming a large $\beta$-barrel pore that results in cell lysis [1]. We have identified a highly conserved structural motif of CDCs that plays a critical role in the prepore-to-pore transition [2]. Furthermore, this motif is also highly conserved in a large, diverse family of uncharacterised proteins from over 220 species, which we have designated the name "CDC-like" (CDCL) protein family [2]. One partner of the CDCL pair, termed CDCL long, consists of four domains: three similar to CDCs and a unique fourth domain. The other partner, CDCL short, possesses three domains, all similar to CDCs. One CDCL pair, referred to as ALY long (ALY $)$ and ALY short $\left(\mathrm{ALY}^{\mathrm{S}}\right)$, originate from the species Elizabethkingia anophelis; an emerging and opportunistic pathogen of unknown virulence and transmission. X-ray crystallography revealed the structure of monomeric $\mathrm{ALY}{ }^{\mathrm{L}}$ consists of characteristic CDC domain $1-3$ structure despite only $22 \%$ identity with the archetype CDC perfringolysin O; however, domain 4 is completely different to the equivalent domain in CDCs that plays a role in sensing cholesterol. In the presence of lipids, $\mathrm{ALY}^{\mathrm{L}}$ and $\mathrm{ALY}^{\mathrm{S}}$ show pore-forming activity and analysis by electron microscopy reveals a large circular oligomeric complex reminiscent of $\mathrm{CDC}$ pore complexes. ALY ${ }^{\mathrm{S}}$ also forms a non-lytic circular oligomer in the absence of $\mathrm{ALY}^{\mathrm{L}}$. Cross-linking mass spectrometry data reveals structural changes between the monomeric and protomeric states, giving insight to the mechanism of pore formation. To determine the atomic structure of ALY pores, cryo-EM single-particle analysis is currently being pursued In summary, we have shown that the ALY toxins share some structural resemblance to CDCs, but in contrast form a two-component pore complex. CDC-like proteins are present in a wide range of bacterial species and are suspected to play key roles in microbial survival and human disease. An understanding of pore formation by ALY may yield new knowledge of Elizabethkingia anophelis virulence, in addition to providing a system that could be applied to biotechnological applications. Our work on ALY provides the first functional and structural insights into this fascinating family of proteins.

[1] Christie MP, Johnstone BA, Tweten RK, Parker MW and Morton CJ (2018) "Cholesterol dependent cytolysins: from water-soluble state to membrane pore", Biophys Rev. 10:1337-1348.

[2] Evans JC, Johnstone BA, Lawrence SL, Morton CJ, Christie MP, Parker MW and Tweten RK (2020), "A Key Motif in the CholesterolDependent Cytolysins Reveals a Large Family of Related Proteins”, mBio, 11(5): e02351-20

Keywords: pore-forming toxin, bacterial proteins, membrane proteins, protein-membrane interactions 